\title{
Two-phase water model in the cellulose network of paper
}

\author{
A. Conti - M. Palombo - A. Parmentier (i) - G. Poggi - P. Baglioni • \\ F. De Luca $(1)$
}

Received: 21 February 2017/ Accepted: 19 May 2017

(C) Springer Science+Business Media Dordrecht 2017

\begin{abstract}
Water diffusion in cellulose was studied via two-phase Kärger model and the propagator method. In addition to ruling out anomalous diffusion, the mean squared displacements obtained at different diffusion times from the Kärger model allowed to characterize the system's phases by their average confining sizes, average connectivity and average apparent diffusion coefficients. The two-phase scheme was confirmed by the propagator method, which has given insights into the confining phasegeometry, found consistent with a parallel-plane arrangement. Final results indicate that water in cellulose is confined in two different types of amorphous domains, one placed at fiber surfaces, the other at fiber cores. This picture fully corresponds to the phenomenological categories so far used to identify
\end{abstract}

\section{A. Conti}

Department of Physics, Sapienza University of Rome,

P.le A. Moro 2, 00185 Rome, Italy

A. Conti

CEA/DRF/I2BM/NeuroSpin, 91191 Gif-sur-Yvette,

France

M. Palombo

CNR ISC UOS, Sapienza University of Rome,

P.le A. Moro 2, 00185 Rome, Italy

M. Palombo

CMIC Department of Computer Science, UCL,

Gower Street, London WC1E 6BT, UK water in cellulose fibers, namely, free and bound water, or freezing and non-freezing water.

Keywords Cellulose $\cdot$ Paper $\cdot$ Water diffusion $\cdot$ PFG NMR · Propagator

\section{Introduction}

Cellulose chains aggregate both in crystalline domains, where chains are highly packed with a well defined unit cell, and amorphous domains (ADs), where chains show little or no order (Nisizawa 1973). The simplest cellulose-chain aggregate is the elementary fibril, which is characterized by a transverse extension of a few nm; elementary fibrils arrange to

\footnotetext{
A. Parmentier

Department of Physics and NAST Center, Tor Vergata University of Rome, via della Ricerca Scientifica 1, 00133 Rome, Italy

G. Poggi · P. Baglioni

Department of Chemistry and CSGI, University of Florence, via della Lastruccia 3, 50019 Sesto Fiorentino, FI, Italy

F. De Luca $(\varangle)$

Department of Physics, Sapienza University of Rome,

P.le A. Moro 2, 00185 Rome, Italy

e-mail: francesco.deluca@roma1.infn.it
} 
form microfibrils, whose transverse extension is a few tens nm (Niskanen 1998). Microfibril bundles form the cellulose fibers, or macrofibrils, whose transverse dimension may be tens $\mu \mathrm{m}$ (Zhao et al. 2007). The supramolecular architecture of cellulose chains is mainly due to a coalescence-like mechanism, which reduces the free energy associated to fibril surfaces, and to Van der Waals interactions, which mostly drive fiber formation. The final arrangement of the fibril structure includes alternating crystalline and amorphous domains along the fibrils, with prevalent crystalline organization (Niskanen 1998; Fengel and Wegener 1984). While crystalline domains are hydrophobic and impenetrable to water, ADs behave as hydrophilic sites where water can interact directly with cellulose chains (Stephens et al. 2008). ADs are also the most vulnerable sites of cellulose chains, since degradation processes, such as acid hydrolysis, are triggered there (Topgaard and Soderman 2001).

Water plays a significant role in the physical properties of cellulose fibers, since it interweaves hydrogen bonds with $\mathrm{OH}$ groups along the chains, therefore modifying fibers' mechanical and electrical properties. Further, water is involved in most degradation processes affecting cellulose (Niskanen 1998). Despite this central role in the properties of cellulose, and therefore in the properties of cellulose-based materials like paper, information about the functional organization of water in cellulose is still lacking, and even today the categorization of water clusters in cellulose is based on their freezing properties (English and MacElroy 2003), or related to generic free and bound water classes (Nakamura et al. 1981).

Recent works on paper, based on low-field NMR relaxation-time and self-diffusion data, suggest that water in cellulose is organized in two phases characterized by two different confinement conditions, both involving ADs (Conti et al. 2014; Lepore et al. 2012). This model accounts well for experimental results, and it is in agreement with the phenomenological characterization that is common in the literature, even though some aspects about phase setting at the fiber scale have still to be specified.

In this work water diffusion in cellulose is studied using both the Kärger model and propagator method in a two-phase system (Price 2009), in which exchange between phases and confining geometries for water diffusion are introduced (Zhao et al. 2007). The approach presented here makes use of the mean squared displacements (MSDs) drawn from the Kärger model at variable diffusion time, in order to get average confining sizes, average connectivity and average apparent diffusion coefficients of water in the two phases (Kaerger et al. 1988). Moreover, the behavior of MSDs versus diffusion time allows to determine whether water's diffusion dynamics is normal or anomalous, which has a considerable impact in assessing confining dimensions and connectivity that characterize the two phases (Conti et al. 2014; Long et al. 2004). Also, NMR data were processed by the propagator method for diffusion (Price 2009), which, apart from providing information directly comparable to results obtained from the Kärger model, allows to retrieve additional insights into the confining phase-geometry.

The two-phase water model here implemented assigns the more mobile water phase to ADs located at fiber surfaces (that is, to ADs of microfibrils at fiber surfaces), while the less mobile one is placed in the ADs of fiber cores (that is, the ADs of microfibrils that are located deep inside the fibers and are nearly isolated from fiber surfaces) (Zhao et al. 2007; Mueller et al. 2000; Schuster et al. 2003).

The samples exploited in this investigation are binder-free cotton-linter paper, whose cellulose fibers have the same structural organization of cellulose in "free" cotton-linter items. Samples were treated at different degrees of hydrolyzation to modify their $\mathrm{AD}$ structure, and therefore the confining condition to which water is subject in the two phases, in order to observe the reliability of the model under different conditions.

\section{Experimental}

Sample preparation

Whatman filter paper (grade 5) composed of raw cotton fibers (minimum $\alpha$-cellulose content: $98 \%$ ) was used for the preparation of our samples. The $S 0$ sample is the untreated one; the $S 1$ and $S 2$ samples were obtained by immersing the filter paper in a $\mathrm{H}_{2} \mathrm{SO}_{4}$ solution $(\mathrm{pH}=1)$. The main difference between these latter two samples concerns the time left to acid hydrolysis to take place: while in $S 2$ the process was stopped $6 \mathrm{~h}$ after the acidification by immersion of the 
sample in MilliQ water (resistivity: $18 \mathrm{M} \Omega$ at $25^{\circ} \mathrm{C}$ ), in $S 1$ the process was not arrested in order to reach the appropriate degree of polymerization (DP). Further details can be found in reference Conti et al. (2014). The DP of the samples was determined by the cuprylethylenediamine method (UNI 8282 1994) using an Ubbelohde viscometer. The values are: $\mathrm{DP}(S 0)=1100 \pm 50, \quad \operatorname{DP}(S 1)=150 \pm 50, \quad$ and $\mathrm{DP}(S 2)=850 \pm 50$.

In order to recognize effects from mere soaking, the SO sample was immersed in distilled water. Before measurements, samples were kept at $22 \pm 1{ }^{\circ} \mathrm{C}$ for $24 \mathrm{~h}$ in a $100 \%$ relative-humidity (RH) environment. Samples were sealed in a plastic film to avoid water loss during NMR measurements.

\section{Diffusion measurements}

Diffusion measurements were performed using a Bruker Avance $300 \mathrm{MHz}$ spectrometer equipped with a gradient unit that generates a maximum gradient intensity of about $1200 \mathrm{G} / \mathrm{cm}$. The sequence used is the PFG-STE (Callaghan 2011), where two G magnetic field gradient pulses of $\delta$ duration $(\delta \cong 1.4 \mathrm{~ms}$ ) are applied within three $90^{\circ}$ radio-frequency pulses. The first two rf pulses are separated by a time interval $\tau_{0} \cong 2.0 \mathrm{~ms}$ and the second pulse has a delay $\Delta$, the diffusion time, with respect to the third. $\Delta$ was changed in different steps to reach a maximum value of $60 \mathrm{~ms}$. For each of the 20 gradient steps, during which the gradient intensity was increased from zero to $1050 \mathrm{G} / \mathrm{cm}, 32$ scans were performed to improve the signal-to-noise ratio. The relaxation recycle delay was fixed to $3 \mathrm{~s}$. The samples of hydrated paper were cut into strips of about $2.5 \times 20 \mathrm{~mm}^{2}$ : after being sealed in a plastic film, they were inserted into the NMR tube for measurements. Measurement temperature was fixed at $22^{\circ} \mathrm{C}$.

The $E(q, \Delta)$ echo amplitude, the dynamic wave vector $q=\gamma \Delta G$, with $\gamma$ the gyromagnetic ratio, and $\Delta$ are related by

$$
E(q, \Delta) \cong E(0, \Delta) e^{-q^{2} D \Delta}
$$

where the condition $\Delta \gg \delta$ has been applied. To avoid relaxation effects the conditions $\tau_{0} \ll T_{2 S}$ and $\Delta \ll$ $T_{1 S}$ were set, where $T_{2 S}$ and $T_{1 S}$ are the shortest longitudinal $\left(T_{1}\right)$ and transverse $\left(T_{2}\right)$ NMR relaxation times, respectively, found for all the samples.

\section{Results and discussion}

Two-phase Kärger model for diffusion

When molecules belong to different exchanging chemo-physical domains, the problem of describing the diffusion-dependent NMR signal is particularly complex. The Kärger model for transport dynamics between two domains (Kaerger et al. 1988) describes the PFG-STE signal as the sum of two echo signals $E(q, \Delta)=E_{1}(q, \Delta)+E_{2}(q, \Delta)$, which are solutions to

$$
\begin{aligned}
\frac{d}{d t} E_{1,2}(q, \Delta)= & -D_{1,2} q^{2} E_{1,2}(q, \Delta)-\frac{E_{1,2}(q, \Delta)}{\tau_{1,2}} \\
& +\frac{E_{2,1}(q, \Delta)}{\tau_{2,1}}
\end{aligned}
$$

where $\tau_{1,2}$ are the molecular residence times in domains 1 and 2, respectively, and $D_{1,2}$ are the selfdiffusion coefficients of water in the same domains, respectively.

Under the hypothesis that the PFG-STE signal of water in paper arises from a coarse-grained average over two different water populations (Conti et al. 2014), the Kärger equation can be solved exactly, giving

$\frac{E(q, \Delta)}{E(0, \Delta)}=p_{A} e^{-q^{2} D_{A} \Delta}+p_{B} e^{-q^{2} D_{B} \Delta}$

where

$$
\begin{aligned}
D_{A, B}= & \frac{1}{2}\left[D_{1}+D_{2}+\frac{1}{q^{2}}\left(\frac{1}{\tau_{1}}+\frac{1}{\tau_{2}}\right)\right. \\
& \left. \pm \sqrt{\left[D_{1}-D_{2}+\frac{1}{q^{2}}\left(\frac{1}{\tau_{1}}-\frac{1}{\tau_{2}}\right)\right]^{2}+\frac{1}{q^{4} \tau_{1} \tau_{2}}}\right]
\end{aligned}
$$

and

$$
p_{A, B}=\frac{ \pm D_{B, A} \mp p_{1} D_{1} \mp p_{2} D_{2}}{D_{B}-D_{A}} .
$$

Here $p_{1}+p_{2}=1$, with $p_{1}$ and $p_{2}$ water-population fractions of phases 1 and 2, respectively. In Fig. 1 the datafits to Eq. (3) for echo decays acquired at the diffusion time of $\Delta=40 \mathrm{~ms}$ are shown for the three samples.

As it can be noticed, signal decays change according to samples, becoming more and more different 
from the trend found for $S 0$ at increasing acidhydrolysis effects.

The mean residence times, found by fit to the Kärger Eq. (3) for water populations in phase 1 and 2 (Fig. 1) at $\Delta=40 \mathrm{~ms}$ are shown in Table 1. Analogous results have been also obtained for the other investigated diffusion times.

From Table 1, it is easy to see how consistently larger than $\Delta=60 \mathrm{~ms}$ - the maximum diffusion time used in the present work-all residence times are. This means that water populations can be considered isolated from each other over all diffusion times spanned during the PFG-STE measurements. A feature of Table 1 that needs to be stressed is the huge difference between the residence time in phase $1\left(\tau_{1}\right)$ and the one in phase $2\left(\tau_{2}\right)$.

In the limit $\tau_{1,2} \gg \Delta$, Eq. (3) is transformed to the simpler expression

$\frac{E(q, \Delta)}{E(0, \Delta)}=p_{1} e^{-q^{2} D_{1} \Delta}+p_{2} e^{-q^{2} D_{2} \Delta}$.

In Eqs. 1, 3 and 6 the diffusion terms have been explicitly written according to a Brownian-like molecular self-diffusion. In the case of anomalous diffusion, the Brownian term $D \Delta$ is transformed to $D_{\alpha} \Delta^{\alpha}$, with $\alpha \neq 1$ and $D_{\alpha}$ the generalized anomalous diffusion coefficient measured in $\frac{m^{2}}{s^{\alpha}}$ units. The echo signal does not distinguish between ordinary and anomalous diffusion, since this difference is made explicit by the $\Delta$-dependence only, while in Eqs. 1, 3 and $6 \Delta$ works as a constant. But, because of the crowding of cellulose chains in the ADs, water

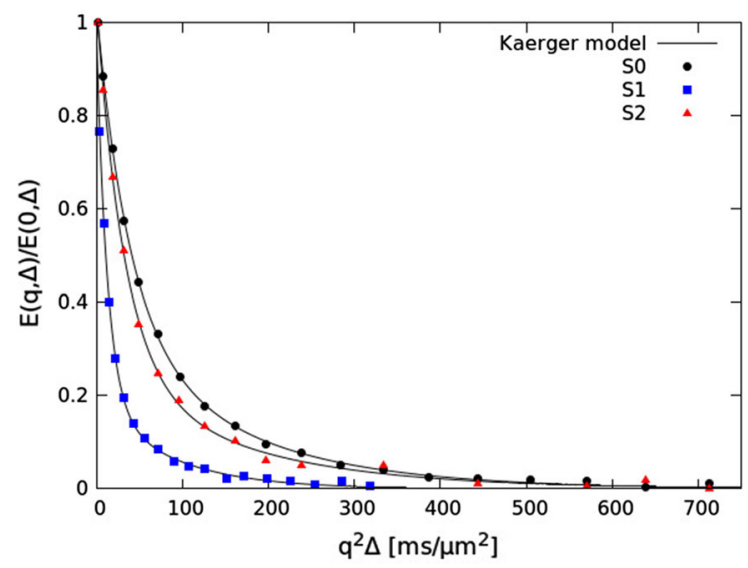

Fig. 1 Datafit to the Kärger model (full line) for echo decays acquired at a diffusion time of $\Delta=40 \mathrm{~ms}$
Table 1 Water-population mean residence times found by datafit to the Kärger Eq. (3) acquired at $\Delta=40 \mathrm{~ms}$ (Fig. 1)

\begin{tabular}{rrrr}
\hline & $S 0$ & $S 1$ & \multicolumn{1}{c}{$S 2$} \\
\hline$\tau_{1}(s)$ & 5.1 & 0.2 & 3.4 \\
$\tau_{2}(s)$ & 28.0 & 6.9 & 27.3 \\
\hline
\end{tabular}

diffusion in this system could be anomalous. Being $<r^{2}(\Delta)>=6 D_{\alpha} \Delta^{\alpha}$ the general expression for the MSD, Eq. (6) can be re-written as

$\frac{E(q, \Delta)}{E(0, \Delta)}=p_{1} e^{-\frac{1}{6} q^{2}<r^{2}(\Delta)>_{1}}+p_{2} e^{-\frac{1}{6} q^{2}<r^{2}(\Delta)>_{2}}$

which is valid for both ordinary and anomalous diffusion (Kimmich 1997). By fitting experimental data acquired at multiple $q$-values and fixed $\Delta$ to Eq. (7), for each $\Delta$ it is possible: (a) to estimate-and, therefore, to assess - whether diffusion in phase 1 and/ or 2 is either anomalous $(\alpha \neq 1)$ or normal $(\alpha=1)$ : in the former case the $\alpha$-value can be related to important features of diffusional dynamics and structural organization of the diffusion patterns (Casieri et al. 2010; Palombo et al. 2013) ; (b) to estimate the ordinary or the anomalous average diffusion coefficient; and c) to evaluate the average confining size for each phase.

The $\left\langle r^{2}(\Delta)>_{1,2}\right.$ data obtained by Eq. (7) are reported in Figs. 2 and 3, respectively, for all of our samples. Since data turn out well fitted to the function $<r^{2}(\Delta)>_{1,2}=6 D_{1,2} \Delta+c_{1,2}$, a Brownian diffusion must be considered over the diffusion time interval taken into account, with $D_{1,2}$ the average apparent diffusion coefficients and $c_{1,2}$ fit constants.

If $D_{1,2}$ are measured at $\Delta$-values larger than the time water needs to diffuse over a distance of the order of the average confining dimension, confinement effects take place (Conti et al. 2014). In this case, if the confining environments (for the sake of simplicity, from now on, we conventionally call them pores) are isolated from each other, $D_{1,2}(\Delta) \rightarrow 0$ and $<r^{2}(\Delta)>_{1,2} \rightarrow c_{1,2}$; if instead $D_{1,2}(\Delta)$ tend to finite values, but lower than the unrestricted diffusion coefficient $D_{U}$, the diffusion coefficient marks a more or less pronounced connectivity within pores (Callaghan 2011). Figure 4 schematically shows these trends in the $\left(\Delta,<r^{2}>\right)$ plane.

In this scheme, the connectivity $C$ between pores can be characterized through the fraction of the 


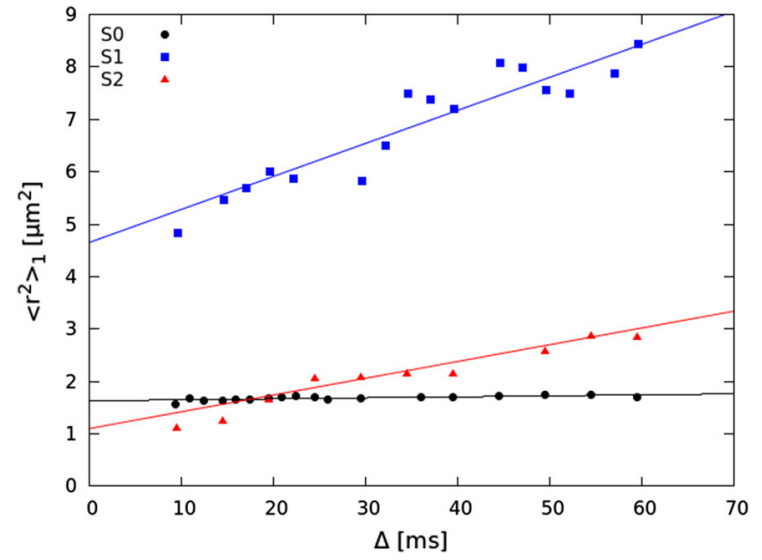

Fig. 2 The MSDs of phase 1 are reported for all samples, for the different investigated diffusion times

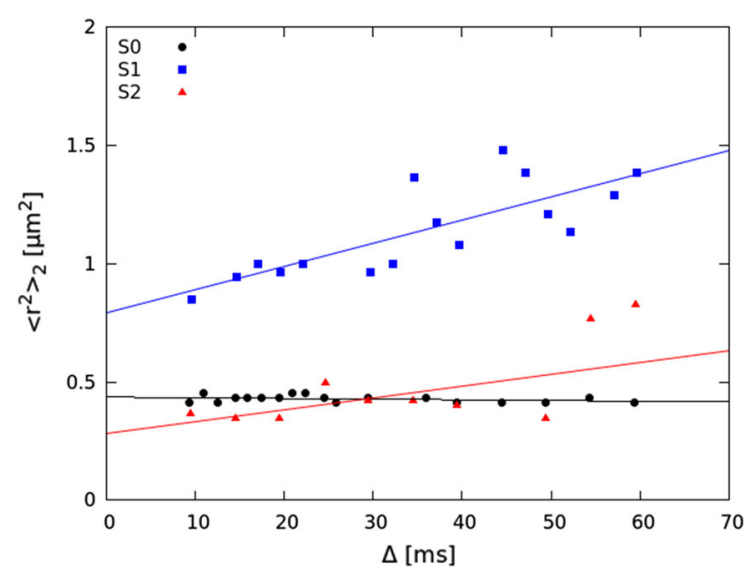

Fig. 3 The MSDs of phase 2 are reported for all samples, for the different investigated diffusion times

unrestricted diffusion slope angle that describes the connected system, that is,

$C_{1,2}=\frac{D_{1,2}}{D_{U}}$.

Equation (8), when $D_{1,2} \rightarrow 0$, returns zero connectivity, while for $D_{1,2} \rightarrow D_{U}$ the connectivity is unity, which coincides with the diffusion limit ratio used to define porous connectivity (Callaghan 2011). Equation (8) implicitly supposes that the slope has a linear behavior respect to the slope angle: this may be considered approximately correct for angles up to about $30^{\circ}$, that is, for a maximum slope of about $\operatorname{tg}\left(30^{\circ}\right)$.

Another possible use of MSDs concerns pore size, and is ruled by $c_{1,2}$ constants. In case of closed pores,

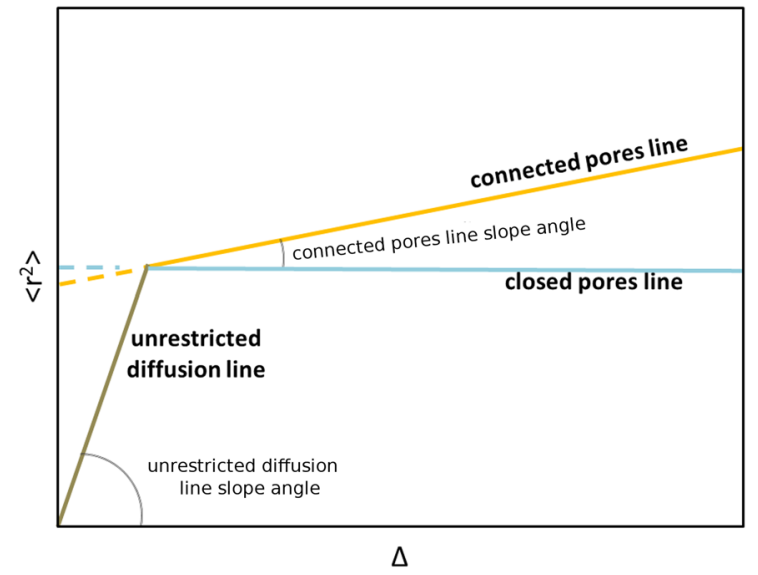

Fig. 4 A scheme of the different MSD behaviors versus $\Delta$ for different confining conditions. The dashed lines show the extension of connected-pore and closed-pore lines to $\Delta=0$. The unrestricted diffusion line marks the $\Delta$-range before the confinement effect starts

the slope is close to zero and the fit line $<r^{2}(\Delta \rightarrow$ $0)>_{1,2}$ returns the average pore dimensions $\sqrt{c_{1,2}}$ with good accuracy (Fig. 4). For connected pores, $\sqrt{c_{1,2}}$ can give information about approximate average pore size, since water would take a few hundreds $\mu$ s to travel a pore diameter of a few $\mu \mathrm{m}$ - as those expected in this case (Conti et al. 2014) —and a few hundreds $\mu$ s may be considered an acceptable $\Delta \rightarrow 0$ limit at the ms scale of the diffusion time. Of course, the condition $c_{1,2} \neq 0$ is a mark of diffusion occurring in a restricted regime (Fig. 4), while $c_{1,2}=0$ is the signature of the unrestricted case.

Figures 2 and 3 clearly show that the $S 0$ sample possesses a pretty closed porous structure, with very limited pore connectivity (Table 2). For this sample, the average apparent diffusion coefficients are, as expected, the smallest ones in both phases, while the average pore sizes, estimated in the limit $\Delta \rightarrow 0$, are $<d>_{1}=1.3 \mu \mathrm{m} \quad$ and $\quad<d>_{2}=0.7 \mu \mathrm{m}$. The $<d>_{1}$-value is a bit smaller than the one obtained by NMR diffraction at a fixed $\Delta$-value (Conti et al. 2014), but the $\langle d\rangle_{1}$ parameter retrieved here is an average value that does not depend on $\Delta$.

The porous structure of phase 1 in the $S 1$ sample, as inferred from Fig. 2, shows a $\langle d\rangle_{1}=2.2 \mu \mathrm{m}$ value, which is about two times that for $S 0$, while its connectivity is about 37 times the one for the same sample (Table 2). The porous structure of phase 1 in the $S 2$ sample shows a $<d>_{1}=1.1 \mu \mathrm{m}$ value, which 
is slightly smaller than in $S 0$, while its connectivity is about 18 times larger (Table 2).

The MSD behavior of phase 2 in $S 1$ and $S 2$ is reported in Fig. 3. The average pore dimension of $S 1$ is $<d>_{2}=0.9 \mu \mathrm{m}$, that is, very close to that of the $S 0$ sample, as well as the $\langle d\rangle_{2}=0.5 \mu \mathrm{m}$ value in the $S 2$ sample. The connectivity of phase 2, both in $S 1$ and $S 2$, significantly decreases with respect to phase 1 (Table 2), even though it is appreciably higher than in $S 0$. Of course, the average apparent diffusion coefficients follow the connectivity behavior. This may suggest that hydrolysis is able to more significantly change connectivity rather than pore size.

In Fig. 5, $p_{1}$ water populations of all samples, obtained from Eq. (7), are reported. $p_{1}$ data have been fitted to the function $p_{1}(\Delta)=m \Delta+p_{01}$, where $p_{01}$ is the steady-state water population of phase 1 . The slopes of $p_{1}$ population versus $\Delta$ are close to zero, which indicates that phase populations are practically constants, while $p_{01}$ is almost the same in all samples. The ratio between water population of phase 1 and 2 is about 3 in each sample (Table 2).

All the above-mentioned results have been summarized in Table 2. The connectivity has been estimated by setting $D_{U}=2.3 \times 10^{-9} \mathrm{~m}^{2} / \mathrm{s}$, which is the diffusion coefficient of bulk water at room temperature. Even though there could be some arbitrariness in choosing this $D_{U}$ value, the comparison between samples is independent of that choice.

It has been shown that the phase 1, i.e., the phase holding more mobile water molecules, adsorbs external water, while the population of phase 2 remains

Table $2 p_{01}$ water population fraction (with $p_{01}+p_{02}=1$ ) estimated in the $\Delta \rightarrow 0$ limit, and average pore sizes estimated from the expression $\left\langle d>_{1,2} \cong \sqrt{c_{1,2}}\right.$, that is, in the limit $<r^{2}(\Delta \rightarrow 0)>_{1,2}$

\begin{tabular}{llcc}
\hline & $S 0$ & $S 1$ & $S 2$ \\
\hline$<d>_{1}(\mu \mathrm{m})$ & 1.3 & 2.2 & 1.1 \\
$<d>_{2}(\mu \mathrm{m})$ & 0.7 & 0.9 & 0.5 \\
$p_{01}$ & 0.75 & 0.74 & 0.77 \\
$D_{1}\left(10^{-12} \mathrm{~m}^{2} / \mathrm{s}\right)$ & 0.3 & 11.0 & 5.4 \\
$D_{2}\left(10^{-12} \mathrm{~m}^{2} / \mathrm{s}\right)$ & 0.1 & 1.6 & 0.8 \\
$C_{1}\left(10^{-4}\right)$ & 1.3 & 47.8 & 23.5 \\
$C_{2}\left(10^{-4}\right)$ & 0.4 & 7.0 & 3.5 \\
\hline
\end{tabular}

The $C_{1,2}$ connectivity parameters (Eq. 8 ) have been estimated by setting $D_{U}=2.3 \times 10^{-9} \mathrm{~m}^{2} / \mathrm{s}$, which is the diffusion coefficient of bulk water at room temperature almost independent of the availability of external water (Conti et al. 2014; Lepore et al. 2012; Proietti et al. 2004). This means that phase 1 and phase 2 have to be associated to different ADs sites. Microfibrils in fibers can be divided into two coarse categories, those belonging to-or close to-fiber surfaces, and microfibrils at fiber cores, respectively (Zhao et al. 2007; Mueller et al. 2000; Schuster et al. 2003). Surface microfibrils possess ADs that are easily attainable by external water, while ADs in core microfibrils are poorly connected to fiber surfaces. It is immediate to assign phase 1 to ADs at fiber surface (AD1s) and phase 2 to ADs at fiber core (AD2s). The average confining dimension $\langle d\rangle_{1}=1.3 \mu \mathrm{m}$ in phase 1 of $S 0$ suggests that the extension of connected ADs at fiber surfaces is at least about $1.3 \mu \mathrm{m}$, while the one internal to fibers extends for about $0.7 \mu \mathrm{m}$ (Table 2). Both of these sizes are consistent with the lateral dimension of fibers, also considering that the confining dimension could be an apparent or effective dimension, since the "medium" in which water diffuses depends on the interlaced effect between the conformation of cellulose chains and the way AD1s and AD2s assemble in fibers (Horner et al. 1995).

This picture is fully confirmed by samples $S 1$ and $S 2$. Hydrolysis breaks cellulose chains in ADs, so changing chain conformation and density (Stephens et al. 2008; Conti et al. 2014; Calvini 2005; Calvini et al. 2008). This tends to enlarge the average pore dimension and to increase connectivity between pores: connectivity is more affected by hydrolysis, since it largely depends on chain conformation and density,

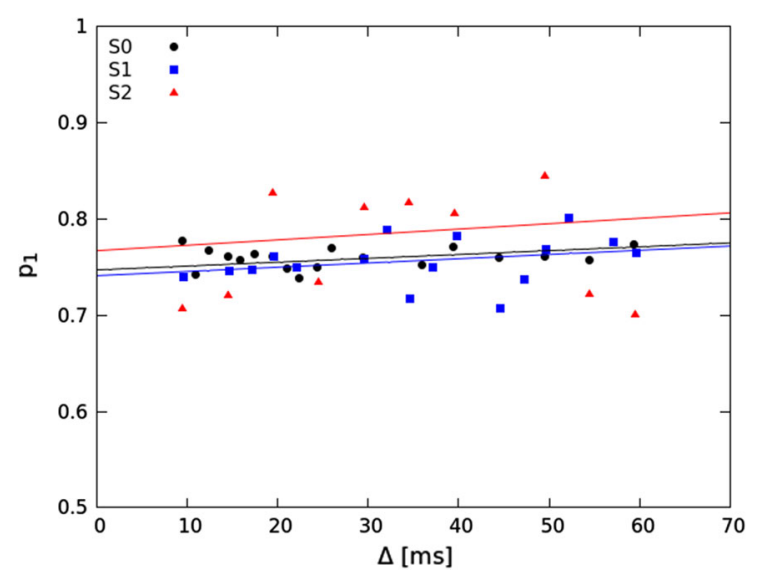

Fig. 5 Behavior of water population in phase 1 for the $S 0, S 1$ and $S 2$ samples 
while pore size is more limited by the extension and geometry of $\mathrm{AD}$ assemblies, which may change mostly due to events able to modify microfibril aggregation. While $S 0$ has pores that are basically isolated from each other, the strong acidification of $S 1$ significantly increases the connectivity, and enlarges pore dimension at fiber surfaces (phase1), which are directly reached by the acid. Conversely, core microfibrils are much less modified (phase 2). This coherently occurs in $S 2$ as well, even though to a less marked extent. In particular, pore size in $S 2$ seems slightly smaller than in $S 0$ : this is not surprising because the light acidification of this sample may modify chain conformation to such an extent that the effective confining dimension may be reduced. Obviously, the average apparent diffusion coefficients in $S 0, S 1$ and $S 2$ follow the behavior of sample connectivity (Table 2).

\section{The propagator method}

As said above, the diffusion of water in cellulose is strictly related to $\mathrm{AD} 1$ or $\mathrm{AD} 2$ organization at the fiber scale. This confining geometry has an anisotropic character, and the anisotropic nature of diffusion is better supported by the diffusion-propagator approach, with appropriate boundary conditions. The diffusion propagator works on the basis of the $P(r \mid r+$ $R, \Delta)$ conditional probability that a molecule at some location $r$ is displaced at $r+R$ in a time $\Delta$. The $E_{1}(q, \Delta)+E_{2}(q, \Delta)$ PFG-STE signal in this case is given by

$$
\begin{aligned}
& E_{1,2}(q, \Delta) \cong F T\left[\frac { 2 } { < d ^ { 2 } > _ { 1 , 2 } } \left[\left|\frac{<d^{2}>_{1,2}}{2}+R_{1,2}\right|-2\left|R_{1,2}\right|\right.\right. \\
& \left.\left.\quad+\left(\frac{<d^{2}>_{1,2}}{2}-R_{1,2}\right) \operatorname{sgn}\left(\frac{<d^{2}>_{1,2}}{2}-R_{1,2}\right)\right]\right]
\end{aligned}
$$

with FT denoting the Fourier transform, and the subscript indicating the corresponding system (or phase). Eq. (9) uses a propagator associated to reflecting planes separated by an average distance $<d>_{1,2}$, which proves a good approximation for the boundary conditions in grouped ADs, after several attempts with different confining geometries (Price 2009). Equation (9) works well with $\Delta$-values longer than the time required by water to diffuse over $<d>_{1,2}$, that is, for $\Delta \gg \frac{<d^{2}>_{1,2}}{D_{U}}$. This condition is respected by all diffusion times spanned in our measurements, for confining distances reported in Table 2. $\left\langle d>_{1,2}\right.$, as well as the population of each phase, can be retrieved from Eq. (9), since the total propagator depends on population fractions. In Fig. 6, $S 0, S 1$ and $S 2$ mean propagators related to phase 2 are reported. As one can see, the profiles of such propagators are fully compatible with MSDs shown in Fig. 3, in particular the one for sample $S 0$, which is practically independent of $\Delta$.

The confining dimensions and the population of the two phases in $S 0, S 1$ and $S 2$ are reported in Table 3. While results for pore size in phase 2 are really close to Kärger data, those for phase 1 are more than a factor 2 larger than data obtained by the same model (Table 2). This is probably due to the more open structure in $\mathrm{AD} 1 \mathrm{~s}$, if compared to $\mathrm{AD} 2 \mathrm{~s}$, which causes the confining geometry used for the propagator to be less effective, not to mention the fact that the propagator is better suited to work on less connected pores.

On the other hand, the relative variation of pore dimensions between sample pairs is fully coherent within the two methods, as it can be seen in Fig. 7 . This suggests that both approaches catch the major features of water organization, even though the role of the effective diffusion paths changes from one method to the other. Further, such differences are, to some extent, an indirect test that the real structure of the two phases for water in cellulose should be very similar to the one described here.

Indeed, on the one hand, coincident confining dimensions for phase 2 from both methods is a sign that the confining geometry adopted for the propagator is well fitted to the AD2 grouping geometry. This confirms that a significant correlation between ADs exists also at the fiber scale, and that the AD2 confining space is more closed, since the propagator works better at measuring its spatial dimension.

On the other hand, the difference between the results from the two approaches confirms that phase 1 is characterized by more open structures than phase 2 , which makes the propagator method to work worse, because this is a technique better suited to treat closed pores. 

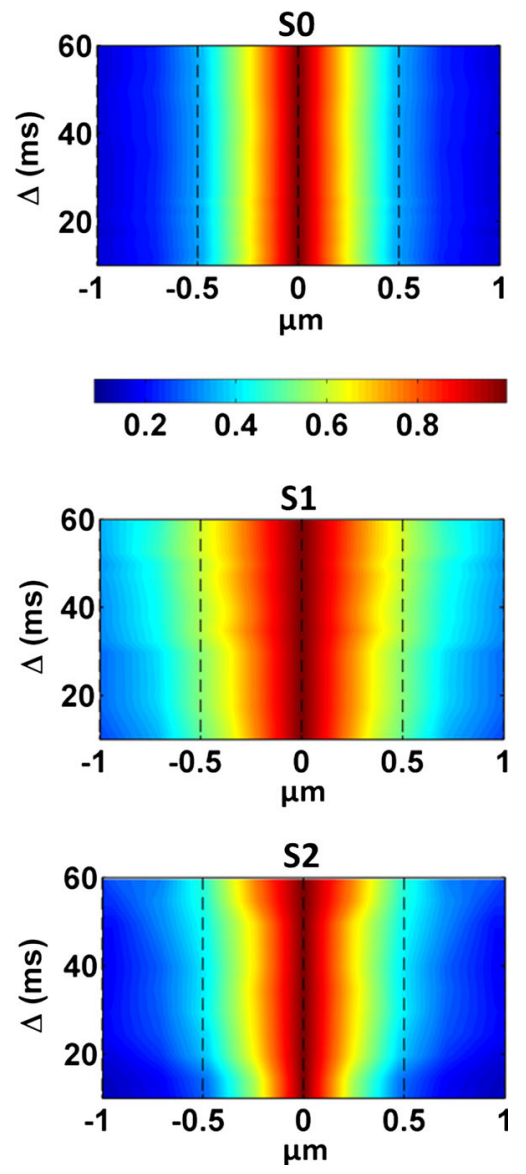

Fig. 6 The mean propagators as a function of the net displacement, $R$ (in $\mu \mathrm{m}$ ), during the diffusion time, $\Delta$ (in $\mathrm{ms}$ ), relative to phase 2 are reported for all samples. The colormap shows the probability density function that a particle in the corresponding sample performs a net displacement $R$ in the given diffusion time $\Delta$

Table 3 Average confining dimensions and $p_{01}$ water populations obtained by the propagator method

\begin{tabular}{llll}
\hline & $S 0$ & $S 1$ & $S 2$ \\
\hline$<d>_{1}(\mu \mathrm{m})$ & 3.3 & 5.5 & 3.5 \\
$<d>_{2}(\mu \mathrm{m})$ & 0.6 & 1.0 & 0.6 \\
$p_{01}$ & 0.65 & 0.74 & 0.67 \\
\hline
\end{tabular}

\section{Conclusions}

Acid hydrolysis has been used to affect the structure of cotton-based paper for the purpose of analyzing the arrangement of water in cellulose. Untreated and hydrolyzed paper samples have been studied via Kärger model and the propagator method, exploiting

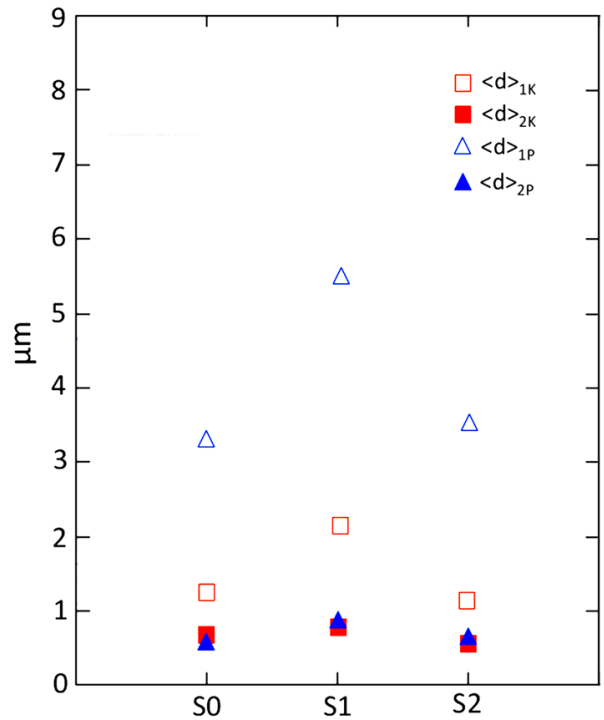

Fig. 7 Comparison between the confining dimensions obtained via Kärger model (subscript $K$ ) and those retrieved by the propagator method (subscript $\mathrm{P}$ )

PFG-STE signals at different diffusion times. By making a comparison between differently hydrolyzed samples $S 0, S 1$ and $S 2$, the arrangement of water in cellulose fibers has been described in some details. Results confirm that water is divided into two main populations arranged in the ADs of microfibrils. The two populations, or phases, have been here associated to different $\mathrm{AD}$ sites: in particular, the population including more mobile molecules has been localized in the ADs at fiber surfaces, that is, in the ADs of microfibrils arranged at, or close to, the fiber surfaces. The propagator method has shown that connectivity is more affected by hydrolysis than pore size, in both $\mathrm{AD}$ phases. Also, when a strong acidification occurs, as in the $S 1$ sample, hydrolysis can enlarge the pore dimension only at fiber surfaces, while core microfibrils are much less modified. The major features of water confinement in both $\mathrm{AD}$ phases have been also tested by the propagator method associated to reflecting planes, proving a good approximation of the boundary conditions both at surfaces and cores of fibrils.

\section{References}

Callaghan P (2011) Translational dynamics and magnetic resonance: principles of pulsed gradient spin echo nmr. Oxford University Press, New York 
Calvini P (2005) The influence of levelling-off degree of polymerisation on the kinetics of cellulose degradation. Cellulose 12:445. doi:10.1007/s10570-005-2206-z

Calvini P, Gorassini A, Merlani A (2008) On the kinetics of cellulose degradation: looking beyond the pseudo zero order rate equation. Cellulose 15:193. doi:10.1007/s10570007-9162-8

Casieri C, Monaco A, De Luca F (2010) Evidence of temperature-induced subdiffusion of water on the micrometer scale in a Nafion membrane. Macromolecules 43(2):638-642. doi:10.1021/ma902323t

Conti A, Poggi G, Baglioni P, De Luca F (2014) On the macromolecular cellulosic network of paper: changes induced by acid hydrolysis studied by NMR diffusometry and relaxometry. Phys Chem Chem Phys 16:8409. doi:10. 1039/C4CP00377B

English N, MacElroy J (2003) Molecular dynamics simulations of microwave heating of water. J Chem Phys 118:1589. doi:10.1063/1.1538595

Fengel D, Wegener G (1984) Wood: chemistry, ultrastructure, reactions. In: Walter de Gruyter. Berlin and New York. doi:10.1002/pol.1985.130231112

Horner A, Milchev A, Argyrakis P (1995) Role of percolation in diffusion on random lattices. Phys Rev E 52:3570. doi:10. 1103/PhysRevE.52.3570

Kaerger J, Pfeifer H, Heink W (1988) Principles and applications of self-diffusion measurements by nuclear magnetic resonance. Adv Magn Res 12:1

Kimmich R (1997) NMR - tomography, diffusometry, relaxometry. Springer, Berlin

Lepore A, Baccaro S, Casieri C, Cemmi A, De Luca F (2012) Role of water in the ageing mechanism of paper. Chem Phys Lett 531:206. doi:10.1016/j.cplett.2012.01.083

Long F, Bagley E, Wilkens J (2004) Anomalous diffusion of acetone into cellulose acetate. J Chem Phys 21:1412. doi:10.1063/1.1699249

Mueller M, Riekel C, Vuong R, Chanzy H (2000) Skin/core micro-structure in viscose rayon fibres analysed by $\mathrm{X}$-ray microbeam and electron diffraction mapping. Polymer 41:2627. doi:10.1016/S0032-3861(99)00433-4

Nakamura K, Hatakeyama T, Hatakeyama H (1981) Studies on bound water of cellulose by differential scanning calorimetry. Text Res J 51:607. doi:10.1177/ 004051758105100909

Nisizawa K (1973) Mode of action of cellulases. J Ferment Technol 51:267

Niskanen K (1998) Paper physics. Fapet Oy, Helsinky

Palombo M, Gabrielli A, Servedio V, Ruocco G, Capuani S (2013) Structural disorder and anomalous diffusion in random packing of spheres. Sci Rep 3:2631

Price W (2009) NMR studies of translational motion. Cambridge University Press, Cambridge

Proietti N, Capitani D, Pedemonte E, Blumich B, Segre A (2004) Monitoring degradation in paper: non-invasive analysis by unilateral NMR, part II. J Magn Reson 170:113. doi:10.1016/j.jmr.2004.06.006

Schuster K, Aldred P, Villa M, Baron M, Loidl R, Biganska O, Patlazhan S, Navard P, Ruef H, Jericha E (2003) Characterising the emerging lyocell fibres structures by ultra small angle neutron scattering (USANS). Lenzinger Ber 82:107

Stephens C, Whitmore P, Morris H, Bier M (2008) Hydrolysis of the amorphous cellulose in cotton-based paper. Biomacromolecules 9:1093. doi:10.1021/bm800049w

Topgaard D, Soderman O (2001) Diffusion of water absorbed in cellulose fibers studied with ${ }^{1} \mathrm{H}-\mathrm{NMR}$. Langmuir 17:2694. doi:10.1021/la000982l

UNI 8282 (1994) cellulose in dilute solutions-determination of limiting viscosity number-method in cupri-ethylenediamine (CED) solution-equivalent to the ISO standard $5351 / 1$

Zhao H, Kwak J, Zhang Z, Brown H, Arey B, Holladay J (2007) Studying cellulose fiber structure by SEM, XRD, NMR and acid hydrolysis. Carbohydr Polym 68:235. doi:10.1016/j. carbpol.2006.12.013 